\title{
Modelo de gestión del talento humano por competencias en la planta de pintura de vehículos CIAUTO, ubicada en Ambato, Ecuador
}

RECIBIDO: 09/05/2019 ACEPTADO: 10/06/2019

\author{
Ángel Mena Reinoso \\ Jorge Freire Miranda ${ }^{2}$ \\ Marco Sinaluisa LoZano ${ }^{3}$ \\ DiEgo SANTILLÁN ESPINOZA ${ }^{4}$
}

\begin{abstract}
RESUMEN
La investigación se desarrolla en la empresa Ciudad del Auto (CIAUTO), en Ambato, Ecuador, de la marca de vehículos Great Wall. Se parte de un análisis bibliográfico, donde se identifican los puestos de trabajo para realizar el diseño de cargos mediante el método Delphi, y se describe las actividades que desempeñará cada trabajador en su puesto. Además, se desarrolla el procedimiento de la gestión del talento humano por competencias en la planta de pintura, por los procesos de admisión, aplicación, compensación, desarrollo, mantenimiento, monitoreo del personal, fortaleciendo el departamento de talento humano. Luego, se realiza un preexperimento bajo la medición del desempeño con base en indicadores de gestión, utilizando el método Likert, al grado de satisfacción, enfocado en sus actitudes, y se obtiene como resultado un incremento de trabajadores satisfechos con su puesto de trabajo de 31,25 a $46,25 \%$. Finalmente, al validar la hipótesis por el método de chi-cuadrado, se puede afirmar que la gestión del talento humano por competencias en el área de pintura de la empresa CIAUTO permite el mejoramiento de sus procesos y procedimientos $y$ aumenta la productividad.
\end{abstract}

Palabras-claves: Productividad; competencias; gestión del talento humano; planta de pintura.

\section{INTRODUCCIÓN}

Con la globalización, los países buscan renovar sus legislaciones y acoplarse a las políticas de las grandes potencias. En Ecuador, a través de la nueva Constitución de 2008, de carácter garantista concerniente a los derechos fundamentales que poseen los ciudadanos y la naturaleza, se ha propiciado la reconstrucción de la base institucional y jurídica de las entidades estatales. Mediante la política estatal implementada en el desarrollo del país bajo el «buen vivir y toda una vida», se busca fortalecer los valores del ser humano, su capacitación y desarrollo tanto intelectual como emocional, pues este debe ser el fin, mas no el objeto de la actividad económica y productiva del Ecuador.

El reto que tienen las empresas, en la actualidad, es disponer de un modelo de gestión del talento humano con una profunda orientación en el trabajo productivo, donde el desarrollo de las organizaciones sea más eficiente. Por ello, habría de tener presente los factores de competitividad que deben intervenir en la permanencia de la empresa en un mercado globalizado: introducción de ciencia y tecnología, generación de valor agregado, asociatividad y capacidad de internacionalizarse, además de un adecuado manejo de la administración y gestión de todo lo referente al talento humano, que definitivamente es el eje central de una empresa. Dichos factores deben ser manejados de forma adecuada para lograr posicionar a las empresas en el mercado y llegar a ser competitivos (Saldarriaga, 2009).

1 Magíster en Matemática Básica y magíster en Informática Empresarial por la Escuela Superior Politécnica de Chimborazo (Riobamba, Ecuador). Actualmente, es docente de la Escuela Superior Politécnica de Chimborazo (Riobamba, Ecuador).

E-mail: angel.mena@espoch.edu.ec

2 Magíster en Ciencias de Ingeniería por la Escuela Superior Politécnica de Chimborazo (Riobamba, Ecuador). Actualmente, es docente titular de dicha institución. E-mail: jfreire@espoch.edu.ec

3 Ingeniero industrial por la Escuela Superior Politécnica de Chimborazo (Riobamba, Ecuador). Actualmente, es consultor independiente. E-mail: marco.sinaluisa@espoch.edu.ec

4 Magíster en Seguridad Industrial y Salud Ocupacional por la Escuela Superior Politécnica de Chimborazo (Riobamba, Ecuador). Actualmente, es docente de dicha institución. E-mail: ivan.santillan@espoch.edu.ec 
En su trabajo, Velásquez (2009) indica que:

Es importante fortalecer la cultura propia de la organización, ya que gracias a ella se crea un grupo de valores, conocimientos y reglas implícitas que rigen el comportamiento diario en el lugar de trabajo y favorece directamente la comunicación entre los miembros de la empresa, creando en los empleados un sentido de pertinencia con el mismo, además con ayuda de una cultura organizacional bien establecida y una estrategia de comunicación interna se obtendrán mejores resultados a nivel interior de la empresa. (p. 32)

Asimismo, Robbins y Judge (2009) señalan que:

La cultura organizacional tiene que ver con la manera en que los empleados perciben las características de la cultura de una organización, no si les gusta. Es decir, es un término descriptivo. Esto es importante debido a que diferencia este concepto del de satisfacción en el trabajo. (p. 552)

Sobre el ambiente laboral y la gestión del talento humano, Velásquez (2009) señala lo siguiente:

El clima laboral es el medio ambiente humano y físico en el que se desarrolla el trabajo cotidiano, influye en la satisfacción y por lo tanto en la productividad. Está relacionado con el "saber hacer» del directivo con los comportamientos de las personas, con su manera de trabajar y de relacionarse, con su interacción con la empresa, con las máquinas que se utilizan y con la propia actividad de cada uno. (p. 37)

Mientras que Chiavenato (2002) dice que:

El Área de Recursos Humanos (ARH) es una de las áreas más afectadas por los recientes cambios que acontecen en el mundo moderno. Las empresas se dieron cuenta de que las personas son el elemento central de su sistema nervioso porque introducen la inteligencia en los negocios y la racionalidad en las decisiones. Tratar a las personas como recursos organizacionales es desperdiciar el talento y la mente productiva. Por tanto, para proporcionar esta nueva visión de las personas, hoy se habla de gestión del talento humano y no de recursos humanos, y ya no se tratan como empleados remunerados en función de la disponibilidad de tiempo para la organización, sino como socios y colaboradores del negocio de la empresa. (p. 23)
Para Tejada (2003), la gestión del talento humano se rige en que «la gerencia del talento humano es la que señala que la dirección y la gestión en la organización se basan en el manejo efectivo de las potencialidades que tienen las personas» (p. 117). Por último, García (2009) indica que:

Una organización adquiere una ventaja competitiva trascendental cuando dedica parte de sus actividades, recursos y tiempo a la formación y gestión eficiente de su talento humano. Por ello, es necesario que el área de la empresa encargada de la gestión humana tenga claramente definidos los procesos y actividades. (p. 164)

La gestión del talento humano por competencias se concibe como una compleja estructura de atributos necesarios para el desempeño en situaciones específicas. Los atributos de carácter, actitudes, valores, el conocimiento de la actividad que realiza, cualquier característica personal que se pueda notar de un modo fiable y que se pueda demostrar entre los trabajadores que mantienen un desempeño excelente o entre los trabajadores eficaces e ineficaces. La idea de este proyecto de investigación es proponer en la empresa Ciudad del Auto (CIAUTO), de Ambato, Ecuador, un nuevo concepto de competencia del ser humano en el campo laboral, la importancia que esto tiene en la organización para ayudar al logro del objetivo de alta productividad, propuesto especialmente en el área de pintura.

En este contexto, en la presente investigación realizada en la planta de pintura de vehículos de la empresa CIAUTO se abordan temáticas de actualidad a partir de una perspectiva teórica-práctica, y se parte desde la planeación estratégica de la gestión del talento humano, la búsqueda de estrategias de reclutamiento, la selección de mano de obra calificada con una correcta descripción y el análisis del cargo y puesto de trabajo. Posteriormente, se surge de la ubicación y de la formación, tomando en cuenta el proceso de rendimiento de los empleados, bajos incentivos y compensaciones, higiene y seguridad industrial y calidad de vida. Todo ello enmarcado en la legislación laboral ecuatoriana, realizando para eso un estudio preexperimental, con un análisis estadístico de un pre y postest.

\section{Competencias}

De acuerdo a la Organización Internacional del Trabajo (OIT), la competencia se define como la capacidad efectiva para llevar a cabo exitosamente una actividad laboral plenamente identificada. Por su parte, la Organización Internacional de Normal- 
ización (ISO) 9000 (2000), en la parte de vocabulario y términos sobre competencia, la conceptualiza como la «habilidad demostrada para aplicar conocimientos y aptitudes» (p. 19). Por lo tanto, competencia es un conjunto de características propias de una persona (actitudes, capacidades, habilidades, destrezas), conocimientos que permiten realizar exitosamente cualquier actividad. En conclusión, la definición del término "competencia» ha sido clave en la concepción del modelo, pues se basa en el reconocimiento de las capacidades personales que implican contenidos ligados a una persona.

\section{Modelo de iceberg}

Spencer y Spencer (1993) definen el modelo de iceberg en dos niveles. En uno de ellos plantean que el conocimiento y las habilidades de las personas son competencias fáciles de observar (visibles) y se desarrollan con capacitaciones; mientras que existe una parte oculta que corresponde a las competencias relativas que tienen que ver con la motivación, las actitudes y los valores que una persona posee y que determinan la personalidad, por lo que esta es la más difícil de modificar o cambiar. Por eso, al momento de la contratación del personal, la formación académica no es suficiente, pues se requiere adicionalmente la valoración de otros aspectos clave que pueden influir positiva o negativamente en el desempeño laboral de acuerdo a los objetivos trazados por la empresa u organización.

\section{Método Delphi}

Esta herramienta ha sido muy utilizada en los estudios de prospectiva; Linstone y Turoff (1975) mencionan que «se puede caracterizar como un método para estructurar un proceso de comunicación grupal, de modo que el proceso sea efectivo para permitir que un grupo de individuos, en su conjunto, enfrente un problema complejo» (p. 3).

Se distingue por el anonimato de la identidad de los individuos que componen el grupo de debate. Además, la iteración y retroalimentación es controlada, sus respuestas se colocan en forma estadística y, sobre todo, se da la heterogeneidad dentro de estos mismos. Asimismo, el método Delphi consta de cinco fases: definición de objetivos, selección de expertos, formación del panel, elaboración y lanzamiento de los cuestionarios y explotación de resultados.

\section{DISEÑO DE LOS CARGOS Y ANÁLISIS DE LOS PUESTOS DE TRABAJO}

Chiavenato (2002) define el cargo como la descripción de las actividades desempeñadas por una per- sona, el análisis y diseño de estos; en cambio, Gómez-Mejía, Balkin y Cardy (2008) mencionan que:

El diseño del puesto de trabajo es el proceso de organización del trabajo en tareas necesarias para llevarlo a cabo. Hay distintos enfoques del diseño del trabajo: la simplificación del trabajo, la ampliación del trabajo, la rotación entre trabajos, el enriquecimiento del trabajo y el diseño del trabajo en función de los equipos. Análisis del puesto de trabajo es el proceso sistemático de recopilación de información para tomar decisiones relativas al trabajo. El análisis del puesto de trabajo identifica tareas, obligaciones y responsabilidades de un determinado trabajo. (p. 99)

En este contexto, se puede concluir en pensar que «el empleado proporciona conocimientos, habilidades, capacidades y destrezas, junto con su aptitud hacia el aprendizaje para alcanzar un desempeño laboral óptimo; la organización debe facilitarle una gestión, donde ese trabajador pueda incrementar esas cualidades para su rendimiento eficiente» (Leal y Sánchez, 2006, p. 542). En ese mismo sentido, Pineda, Pérez y Arango (2012) señalan que «el rendimiento por contrato fue el indicador base para medir la productividad. Un contrato es una serie de máquinas asignadas a un trabajador; el indicador de rendimiento es la relación entre la producción real y la esperada» (p. 38). Sherman, Bohlander y Snell (1999), así como Rue y Byars (2000) mencionan que en el diseño se define cómo se van a realizar las funciones, quién y dónde se llevarán a cabo las labores que debe realizar una persona o un grupo en su puesto de trabajo.

\section{Modelo de gestión del talento humano}

La gestión del talento humano es definida por Chiavenato (2009) como «el área que construye talentos por medio de un conjunto integrado de procesos, y que cuida al capital humano de las organizaciones, dado que es el elemento fundamental de su capital intelectual y la base de su éxito» (p. 9). Por su parte, Eslava (2004) afirma que esta gestión del talento humano:

Es un enfoque estratégico de dirección cuyo objetivo es obtener la máxima creación de valor para la organización, a través de un conjunto de acciones dirigidas a disponer en todo momento del nivel de conocimiento, capacidades y habilidades en la obtención de los resultados necesarios para ser competitivos en el entorno actual y futuro. (p. 86) 
Otro de los puntos es desarrollado por Solano (2010) cuando indica que los modelos de gestión del talento humano:

Establecen los requerimientos mínimos que, al ser cubiertos, pueden conducir a la satisfacción de los clientes, con la gestión de la calidad, al bienestar de las personas en el sitio de trabajo, con la gestión de la seguridad y salud, a reducir la contaminación, con la implementación de un sistema de gestión ambiental. (p. 84)

De esta forma, el contenido mínimo del modelo de gestión es diagnóstico inicial, requisitos legales, establecimiento de aspectos significativos, establecimiento de áreas de trabajo, definición de responsables, instrucciones de trabajo, control de acciones correctivas, procedimientos y lineamientos.

\section{Medición de las competencias}

En relación al desarrollo de las competencias, estas se pueden medir antes y después de la implementación de planes de desarrollo; y, de tal forma, comparar los resultados y sacar las conclusiones adecuadas. Así, una de las metodologías es la que propone Alles (2005), quien plantea, primero, medir las competencias deseadas o requeridas, para luego evaluar y medir al primer año; y, después, al segundo año, si las competencias se acercan al perfil requerido, las competencias habrán sido eficaces.

La demostración del cambio de comportamiento se calcula con:

$$
C_{A p}=\frac{\operatorname{Ap}(\mathrm{d})}{\operatorname{Ap}(\mathrm{a})}
$$

Donde:

$C_{A p}=$ cambio observable en aptitudes como consecuencia de la formación

$A p(d)=$ aptitudes demostradas después de la formación en el trabajo

$\mathrm{Ap}(\mathrm{a})=$ nivel de aptitud existente antes de la formación, usando los mismos criterios de medición

La información existente al interior de los cálculos se obtiene y recopila mediante cuestionarios, entrevistas y observaciones.

\section{METODOLOGÍA}

Esta investigación es de tipo preexperimental, pues según Hernández, Fernández y Baptista (2014) sirve como estudio exploratorio con preprueba/posprueba en un solo grupo; además, es descriptiva, aplicativa y de campo, donde con un procedimiento metódico de recolección, análisis y presentación formal de datos, apoyada en una técnica de recolección directa de la empresa CIAUTO, de la ciudad de Ambato (Ecuador), se establece contacto con las estaciones de trabajo de electroforesis (método de pintura ELPO) para analizar el proceso de sellado y primer proceso de pintura top coat. Asimismo, se utilizó el método Delphi para recopilar los juicios emitidos por el grupo de expertos de la empresa: gerente general, coordinador de talento humano, líder del área de soldadura, líder del área de pintura, coordinador del área financiera y operarios, todo esto a través de un cuestionario y entrevista.

\section{Procedimiento de la gestión del talento humano por competencias}

La gestión del talento humano por competencias en la planta de pintura de la empresa CIAUTO de Ambato, Ecuador, está constituida por los subsistemas: reclutamiento y selección, capacitación y evaluación del desempeño; véase la figura 1.

\section{Documentos y registros asociados}

Se analizaron los documentos que dispone la empresa para efectuar la selección, contratación, capacitación y evaluación del personal que va a efectuar las actividades en la estación de trabajo, pues estos permiten realizar los procesos y subprocesos de la gestión del talento humano, los cuales son:

- [SOP-06-PL-01] Plan de capacitación.

- $\quad$ [SOP-06-FR-01] Formato de perfil del cargo.

- [SOP-06-FR-02] Formato de solicitud de empleo y entrevista.

- [SOP-06-FR-03] Formato de requisitos de documentos para el file del empleado.

- $\quad$ [SOP-06-FR-04] Formato de inducción para el puesto de trabajo.

- [SOP-06-FR-05] Formato de registro de capacitación.

- [SOP-06-FR-06] Formato de evaluación del personal (3 meses). 


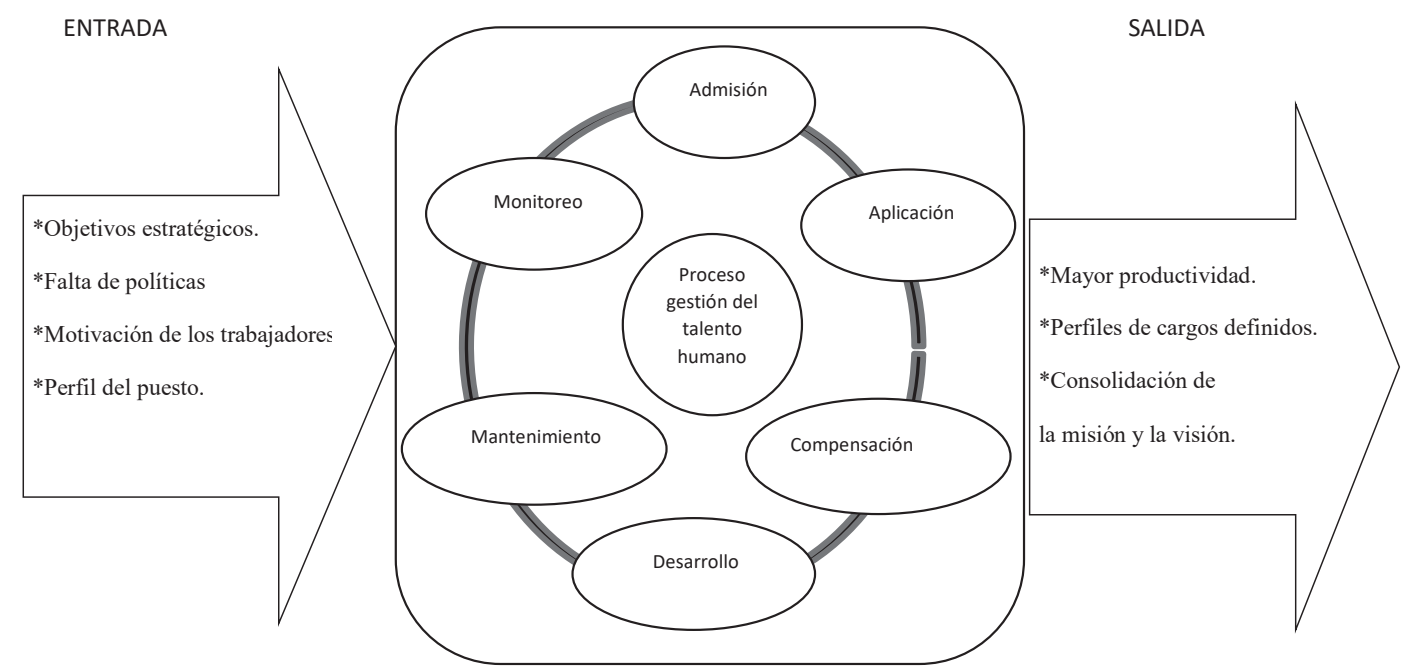

Figura 1. Gestión del talento humano por competencias.

Fuente: Elaboración propia.

- [SOP-06-FR-07] Formato de evaluación del personal (12 meses).

- [SOP-06-FR-08] Formato evaluación de desempeño por capacitaciones.

\section{Perfil del puesto}

La identificación de los perfiles se realiza con el modelo de iceberg, en el cual se plantean dos grandes bloques. El primero de ellos es reconocer las capacidades fáciles (habilidades, conocimientos de ciencias básicas, materiales y pintura), además se plantea una encuesta estructurada no disfrazada con preguntas abiertas para conocer las actividades de cada cargo. El segundo bloque aborda las capacidades difíciles de detectar, por ello, se aplican los exámenes de competencias y actitudes que presenta el trabajador hacia la empresa.

\section{Responsabilidad y autoridad}

El responsable de la implementación, mantenimiento y mejora del presente trabajo es el coordinador de talento humano. También es responsable de desarrollar y coordinar las actividades de reclutamiento y selección, capacitación, evaluación del desempeño y de la eficacia de las capacitaciones del personal de la organización.

\section{Encuesta}

La encuesta está enfocada en medir las actitudes de los trabajadores en formato de respuesta tipo, de acuerdo al método Likert, tal como se aprecia en la tabla 1.

\section{CUESTIONARIO}

Objetivo: identificar la actitud o comportamiento que presentan los trabajadores en la ejecución de sus actividades laborales.

Marque con una $\mathbf{X}$ la respuesta que considere correcta de acuerdo a la pregunta $(5=$ muy satisfecho, 4 = satisfecho, $3=$ medianamente satisfecho, 2 = insatisfecho, 1 = muy insatisfecho).

El método empleado en la investigación es deductivo. La información de los instrumentos de evaluación fue tabulada con apoyo de los softwares Microsoft Excel y SPSS Statistics 21, mientras que la hipótesis se comprobó con el método chi-cuadrado, con los datos del antes y después.

\section{Población y muestra}

La población tomada en cuenta para la investigación fue constituida por ocho trabajadores, que es el total de personas que laboran en el área de pintura de la empresa CIAUTO.

La encuesta fue sometida a una estadística de fiabilidad obteniéndose un alfa de Cronbach de 0.627 , lo que significa que el test aplicado tiene un aceptable nivel de fiabilidad.

\section{RESULTADOS Y DISCUSIÓN}

Se realizaron las encuestas para obtener los parámetros que permitan mejorar las metas de la empresa. Para lograr una correcta gestión del talento 
Tabla 1. Encuesta para medir actitudes de los trabajadores.

\begin{tabular}{|c|c|c|c|c|c|}
\hline PREGUNTA & 1 & 2 & 3 & 4 & 5 \\
\hline ¿Cómo se encuentra en su puesto de trabajo? & & & & & \\
\hline ¿Cómo califica la atmósfera del lugar donde usted labora? & & & & & \\
\hline ¿Está satisfecho con la actitud que presenta su jefe inmediato? & & & & & \\
\hline ¿Qué tan satisfecho se siente con el trato que le brinda la empresa? & & & & & \\
\hline $\begin{array}{l}\text { ¿Está satisfecho con las condiciones de trabajo que le otorga la empresa para la re } \\
\text { de las tareas? }\end{array}$ & & & & & \\
\hline ¿Está satisfecho con la imparcialidad de sus superiores? & & & & & \\
\hline ¿Cómo se siente con la libertad que le dan para realizar el trabajo? & & & & & \\
\hline ¿Está satisfecho con la atención y el trato que se le otorga al cliente? & & & & & \\
\hline ¿Está satisfecho con el trato que recibe dentro de la empresa? & & & & & \\
\hline ¿Qué tan satisfecho está con los procesos y procedimientos dentro de la empresa? & & & & & \\
\hline
\end{tabular}

Fuente: elaboración propia.

humano, se midió cada proceso productivo, cuyo resultado está presente en la tabla 2.

Tabla 2. Índice de productividad antes de la implementación de mejoras.

\begin{tabular}{|c|c|c|c|}
\hline Día & $\begin{array}{c}\text { Producción } \\
\text { (vehículos) }\end{array}$ & $\begin{array}{c}\text { Tiempo } \\
\text { (horas) }\end{array}$ & $\begin{array}{c}\text { Índice de } \\
\text { productividad }\end{array}$ \\
\hline 1 & 4 & 7,50 & 0,533 \\
\hline 2 & 5 & 8,00 & 0,625 \\
\hline 3 & 4 & 8,00 & 0,500 \\
\hline 4 & 5 & 8,00 & 0,625 \\
\hline 5 & 4 & 7,00 & 0,571 \\
\hline 6 & 4 & 7,50 & 0,533 \\
\hline 7 & 4 & 7,75 & 0,516 \\
\hline 8 & 5 & 7,50 & 0,667 \\
\hline 9 & 5 & 8,00 & 0,625 \\
\hline 10 & 5 & 8,00 & 0,625 \\
\hline 11 & 5 & 8,00 & 0,625 \\
\hline 12 & 5 & 7,75 & 0,645 \\
\hline 13 & 4 & 7,25 & 0,552 \\
\hline 14 & 5 & 8,25 & 0,606 \\
\hline 15 & 4 & 7,50 & 0,533 \\
\hline \multicolumn{2}{|c|}{ Promedio índice de productividad } & 0,585 \\
\hline
\end{tabular}

Fuente: elaboración propia.

De la tabla 2 se aprecia que el índice de productividad es de 0,585 vehículos por hora, de esta forma se determinó que cada $1 \mathrm{~h} 42$ min se pinta un vehículo. Por lo tanto, la productividad es de 4,68 vehículos por día, considerando un día laborable de ocho horas.
Posteriormente, se efectuó una encuesta para identificar cómo se encuentra la actitud de los trabajadores en su puesto de trabajo, para lo cual se empleó la tabla 1 . Se realizó la misma el 15 de noviembre del 2016, cuyo resultado mostró que el $38,75 \%$ se encuentran medianamente satisfechos. Véase la siguiente figura:

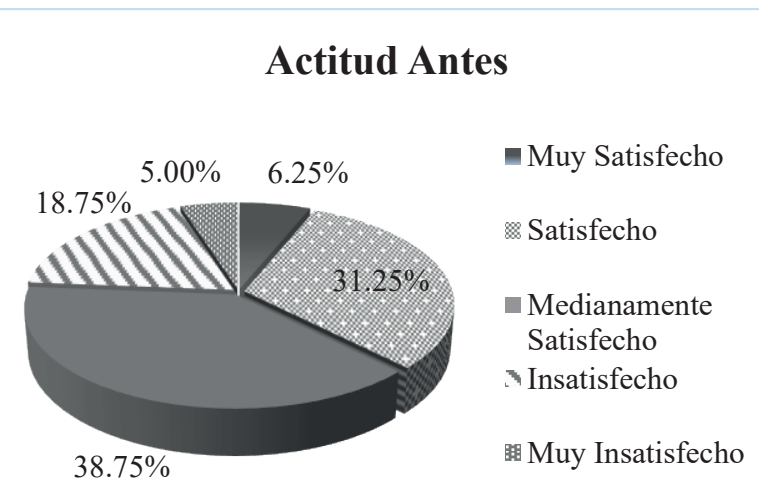

Figura 2. Frecuencia actitudes al 15 de noviembre del 2016.

Fuente: elaboración propia.

Pese a ser una investigación de tipo preexperimental, después de tres meses tras haber aplicado las mejoras, se realizó la encuesta a los empleados de la planta de pintura para poder determinar los cambios en las actitudes de los empleados hacia la empresa. Así, se demostró que el $46,25 \%$ se encontraron satisfechos con su puesto de trabajo y condiciones. Véase la siguiente figura: 


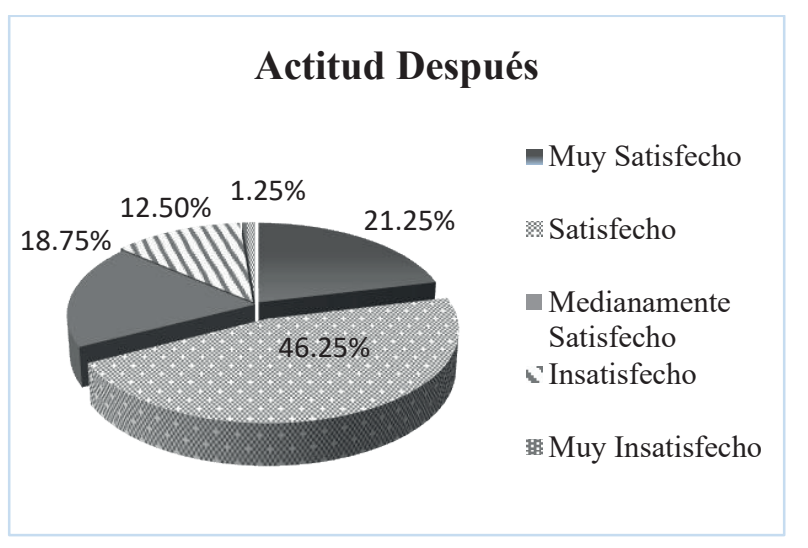

Figura 3. Frecuencia actitudes al 1 de marzo del 2017 (postest).

Fuente: elaboración propia.

Se midió el índice de productividad tras las mejoras, y se obtuvieron los datos que se muestran en la tabla 3.

Tabla 3. Índice de productividad después de la implementación de mejoras.

\begin{tabular}{|c|c|c|c|}
\hline Día & $\begin{array}{c}\text { Producción } \\
\text { (vehículos) }\end{array}$ & $\begin{array}{c}\text { Tiempo (ho- } \\
\text { ras) }\end{array}$ & $\begin{array}{c}\text { Índice de } \\
\text { productividad }\end{array}$ \\
\hline 1 & 7 & 8 & 0,875 \\
\hline 2 & 7 & 8 & 0,875 \\
\hline 3 & 8 & 8 & 1,000 \\
\hline 4 & 8 & 8 & 1,000 \\
\hline 5 & 8 & 8 & 1,000 \\
\hline 6 & 8 & 8 & 1,000 \\
\hline 7 & 7 & 8 & 0,875 \\
\hline 8 & 7 & 8 & 0,875 \\
\hline 9 & 7 & 8 & 0,875 \\
\hline 10 & 8 & 8 & 1,000 \\
\hline 11 & 7 & 8 & 0,875 \\
\hline 12 & 7 & 8 & 0,875 \\
\hline 13 & 7 & 8 & 0,875 \\
\hline 14 & 7 & 8 & 0,875 \\
\hline 15 & 8 & 8 & 1,000 \\
\hline \multicolumn{2}{|l|}{ Promedio índice de productividad } & 0,925 \\
\hline
\end{tabular}

Fuente: elaboración propia.

En la tabla 3 se aprecia que el índice de productividad es de 0,925 vehículos pintados por hora y de 7,4 vehículos por día, considerando ocho horas laborales.

De acuerdo a las figuras 3 y 4 de las actitudes antes y después, se observa que existe un cambio en actitud de los trabajadores hacia la empresa, ya que se ha incrementado el número de trabajadores que se sienten muy satisfechos de 6,25 a $21,25 \%$ y satisfechos de 31,25 a 46,25\%. Asimismo, también hay una reducción de las personas que se sienten medianamente satisfechos de 38,75 a $18,75 \%$, insatisfechos de 18,75 a $12,5 \%$ y muy insatisfechos de 5 a $1,25 \%$.

Para verificar el cambio en el comportamiento de los trabajadores, en especial con el caso «satisfactorio», se utiliza la ecuación (1), lo que da como resultado 1,48 (mayor a 1 se considera una transformación positiva en las actitudes). Esto se aprecia también al observar las tablas 2 y 3 , en las que el promedio índice de productividad pasó de 0,585 antes de aplicar la gestión del talento humano por competencias-a 0,925.

Para determinar factiblemente que se cumplió con el objetivo planteado, se utilizaron la hipótesis nula $(\mathrm{Ho})$ y la hipótesis alternativa $(\mathrm{Ha})$.

Ho: la gestión del talento humano por competencias, en el área de pintura de la empresa CIAUTO, no permite el mejoramiento de sus procesos y procedimientos y disminuye la productividad.

Ha: la gestión del talento humano por competencias, en el área de pintura de la empresa CIAUTO, permite el mejoramiento de sus procesos y procedimientos y aumenta la productividad.

Para la comprobación, se planteó la siguiente interrogante: Ho: Py = Px; Ha: Py > Px, para ello se tabularon los datos en tabla 4.

Con un margen de error del $5 \%$ y $5^{\circ}$ de libertad se obtiene el chi-cuadrado tabulado, que es de 11,07. Luego, con el uso del software SPSS Statistics 21 se comprobó la hipótesis. Véase la tabla 5.

Finalmente, en la tabla 5 se observa que el chi-cuadrado calculado es $17,55(p<0,05)$, con lo cual se rechaza la hipótesis nula y se acepta la alterna (Ha); es decir, de acuerdo a la investigación que se realiza, la gestión del talento humano por competencias en el área de pintura de la empresa CIAUTO permite el mejoramiento de sus procesos y procedimientos y aumenta la productividad.

\section{CONCLUSIONES}

1. Se define el modelo de gestión del talento humano por competencias en el área de pintura de la empresa de vehículos CIAUTO, de la ciudad de Ambato (Ecuador), por los procesos de admisión, preservación y monitoreo del personal. Por ello, se realiza el análisis y diseño de 
Tabla 4. Frecuencias observadas y frecuencias esperadas.

\begin{tabular}{|l|c|c|c|c|}
\hline \multirow{2}{*}{ Variable } & \multicolumn{2}{|c|}{ Frecuencias observadas } & \multicolumn{2}{c|}{ Frecuencias esperadas } \\
\cline { 2 - 5 } & Antes (Px) & Después (Py) & Antes & Después \\
\hline Muy satisfecho & 5 & 17 & 10,83 & 11,17 \\
\hline Satisfecho & 25 & 37 & 30,51 & 31,49 \\
\hline Medianamente satisfecho & 31 & 15 & 22,64 & 23,36 \\
\hline Insatisfecho & 15 & 10 & 12,30 & 12,70 \\
\hline Muy insatisfecho & 4 & 1 & 2,46 & 2,54 \\
\hline Productividad & 4,68 & 7,4 & 5,95 & 6,14 \\
\hline Subtotal & 84,68 & 87,4 & & \\
\hline
\end{tabular}

Fuente: elaboración propia.

Tabla 5. Prueba de chi-cuadrado.

\begin{tabular}{|l|c|c|c|}
\hline & Valor & Grados de libertad & Sig. asintótica (bilateral) \\
\hline Chi-cuadrado de Pearson & $17,546^{\mathrm{a}}$ & 5 & 0,004 \\
\hline Razón de verosimilitudes & 18,183 & 5 & 0,003 \\
\hline Asociación lineal por lineal & 11,265 & 1 & 0,001 \\
\hline N. ${ }^{\circ}$ de casos válidos & 172 & & \\
\hline
\end{tabular}

Fuente: elaboración propia.

a. Dos casillas (16,7\%) tienen una frecuencia esperada inferior a 5. La frecuencia mínima esperada es 2,47.

los cargos utilizando el método Delphi y se describen las actividades que desempeñará cada persona en su puesto de trabajo. Así, para su medición, se debe tomar en cuenta el antes y después de la implementación de los planes. Luego, se debe evaluarlos de acuerdo al método Likert, aplicando estrategias de medición de desempeño basadas en indicadores de gestión según la función del análisis de las competencias.

2. Se determina el procedimiento de la gestión del talento humano por competencias en el área de pintura de la empresa de vehículos CIAUTO por los procesos de admisión, aplicación, compensación, desarrollo, mantenimiento y monitoreo del personal; y, con el análisis de los requerimientos de la planta, sus responsables de la implementación, el mantenimiento y la mejora de los procesos, se establece documentos y registros asociados.

3. Con el procedimiento de gestión del talento por competencias en el área de pintura de la empresa de vehículos CIAUTO se fortalece el departamento de talento humano como un medio para alcanzar eficacia y eficiencia, generados de una mayor competitividad de recursos humanos, que establece el proceso y garantiza la capacitación del personal, y con una adecuada definición del puesto de trabajo se logra una correcta selección y ubicación adecuada del personal y, por consecuencia, en su retención, con el objetivo de llevar a la empresa al éxito.

4. A pesar de que es una investigación de tipo preexperimental, se utiliza el análisis estadístico; por lo que, al aplicar la medición de competencias con un pre y postest, se obtiene un valor de 1,48. Luego de reconocer que existe una transformación positiva en las actitudes, se tiene trabajadores motivados, eficientes, con una capacitación permanente y renumerados según su desempeño, destrezas y habilidades, con lo que se obtiene un aumento de la productividad de 4,68 vehículos a 7,4 por día.

5. La implementación de la política de gestión del talento humano por competencias en el área de pintura de la empresa CIAUTO permite el mejoramiento de sus procesos y procedimientos y aumenta la productividad.

\section{REFERENCIAS BIBLIOGRÁFICAS}

[1] Alles, M. (2005). Desempeño porcompetencias. Evaluación $360^{\circ}$. Buenos Aires, Argentina: Ediciones Granica. Recuperado de https:// www.academia.edu/22501484/Alles-MarthaDesempeño-por-competencias-de-360. 
[2] Chiavenato, I. (2002). Gestión del talento humano. Bogotá, Colombia: McGraw-Hill.

[3] Chiavenato, I. (2009). Gestión del talento humano. México, D. F., México: McGraw-Hill.

[4] Eslava, E. (2004). Gestión del talento humano en las organizaciones. Recuperado de https:// es.scribd.com/document/114171987/GestionTalento-Humano-Organizaciones-Libro.

[5] García, M. (2009). Los macro-procesos: un nuevo enfoque en el estudio de la gestión humana. Pensamiento y Gestión, (27), 162-200.

[6] Gómez-Mejía, L.; Balkin, D. y Cardy, R. (2008). Gestión de recursos humanos. Madrid, España: Pearson Educación S. A.

[7] Hernández, R.; Fernández, C. y Baptista, M. (2014). Metodología de la investigación. México, D. F., México: McGraw-Hill.

[8] Leal, R. y Sánchez, N. (2006). Competencias profesionales del personal operativo de las empresas automotrices para su eficiente desempeño laboral. Revista Venezolana de Ciencias Sociales, 10(2), 541-560.

[9] Linstone, H. A. y Turoff, M. (Eds.) (1975). The Delphi method. Techniques and applications. Recuperado de https://web.njit.edu/ turoff/ pubs/delphibook/delphibook.pdf.

[10] Organización Internacional de Normalización (ISO) 9000 (2000). Norma internacional. Sistemas de gestión de la calidad: conceptos $y$ vocabulario. Recuperado de https:// gestiondecalidadmpn.files.wordpress. com/2012/02/iso-9000-2000-sistemasde-gestic $3 b 3 n$-de-la-calidad-conceptos-yvocabulario.pdf.

[11] Pineda, U.; Pérez, G. y Arango, M. (2012). Medición del impacto de las competencias laborales en la productividad de los procesos: caso de una empresa manufacturera. Innovar. Revista de Ciencias Administrativas y Sociales,
22(45), 37-50. Recuperado de https://www. redalyc.org/pdf/818/81824864004.pdf.

[12] Robbins, S. y Judge, T. (2009). Comportamiento organizacional. México, D. F., México: Pearson Educación.

[13] Rue, L. y Byars, L. (2000). Management, Skills and Applications. Boston, EE: UU: Irin / McGraw-Hill.

[14] Saldarriaga, J. G. (2009). La responsabilidad social aplicada como estrategia de gestión humana. Recuperado de http://www.ascolfa. edu.co/web/archivos/conferencia2009/ memorias/MemoriasCladea2009/upac01_ submission_411.pdf.

[15] Sherman, A.; Bohlander, G. y Snell, S. (1999). Administración de recursos humanos. México, D. F., México: Thomson Editores.

[16] Solano, P. (2010). Modelos de gestión ambiental aplicados en la Compañía Ecuatoriana de Caucho S. A. (Tesis de maestría). Universidad de Cuenca, Cuenca.

[17] Spencer, L. y Spencer, P. (1993). Competence at work: models for superior performance. Nueva York, EE. UU.: John Wiley \& Sons Inc.

[18] Tejada, A. (2003). Los modelos actuales de gestión en las organizaciones. Gestión del talento, gestión del conocimiento y gestión por competencias. Psicología desde el Caribe, (12), 115-133. Recuperado de https://www. redalyc.org/pdf/213/21301208.pdf.

[19] Velásquez, M. (2009). Propuesta de planeación estratégica para la gestión del talento humano de la sección agencia de viajes del departamento comercial de la subdirección de recreación de CAFAM. (Tesis de licenciatura). Universidad La Salle, Arequipa. 


\title{
Competency-based Human Management Model at the CIAUTO vehicle painting department in Ambato, Ecuador
}

\author{
Ángel Mena Reinoso ${ }^{1}$ \\ JoRGE FrEIRE MIRANDA ${ }^{2}$ \\ Marco Sinaluisa Lozano ${ }^{3}$ \\ Diego SANTILLÁn EsPinOZA
}

Received: 09/05/2019 Accepted: 10/06/2019

\begin{abstract}
This study was conducted at the Ciudad del Auto (CIAUTO) company in Ambato, Ecuador, owned by motor vehicle company Great Wall. First, a literature review was conducted where jobs were identified in order to design roles using the Delphi method; the activities performed by each worker in their role were also described. In addition, the process of competency-based human talent management in the painting department was developed using the processes of admission, application, compensation, development, maintenance, personnel monitoring and strengthening the human talent department. Subsequently, a pre-experiment performance measurement based on management indicators was conducted using the Likert Scale to assess the degree of employee satisfaction, focused on attitudes, which resulted in an increase in job satisfaction from $31.25 \%$ to $46.25 \%$. Finally, upon validation of the hypothesis using the Chi-square test, it can be said that the competency-based human management in the CIAUTO painting department improves its processes and procedures and increases productivity.
\end{abstract}

Keywords: Productivity; skills; human talent management; painting plant

\section{INTRODUCTION}

As a result of globalization, countries seek to overhaul their laws and adapt their policies to those of powerful countries. In Ecuador, reconstruction of the institutional and legal foundation of government entities has been promoted in virtue of the new Constitution of 2008, which guarantees the fundamental rights of citizens and nature. Through state policy implemented in the National Development Plan based on the "buen vivir y toda una vida" [good living and a lifetime] vision, the country looks to strengthen human values, training and growth both intellectually and emotionally, as this should be the purpose of Ecuador's economic activities.

The challenge for companies today is to provide a human talent management model that is deeply focused on productive work, where organizational development can be more efficient. Therefore, the competitiveness factors involved in the permanence of the company in a globalized market should be considered. These factors are: introduction of science and technology, generation of added value, partnership, ability to internationalize as well as adequate human talent management-which is the absolute core of a company. These factors must be properly managed in order to position companies in the market and become competitive (Saldarriaga, 2009).

Master in Basic Mathematics and Master in Business Informatics from the Escuela Superior Politécnica de Chimborazo (Riobamba, Ecuador). Currently working as Professor at Escuela Superior Politécnica de Chimborazo (Riobamba, Ecuador).

E-mail: angel.mena@espoch.edu.ec

2 Master in Engineering Sciences from the Escuela Superior Politécnica de Chimborazo (Riobamba, Ecuador). Currently working as Professor at said institution. E-mail: jfreire@espoch.edu.ec

3 Industrial Engineer from the Escuela Superior Politécnica de Chimborazo (Riobamba, Ecuador). Currently working as an independent consultant. E-mail: marco.sinaluisa@espoch.edu.ec

4 Master in Industrial Safety and Occupational Health from the Escuela Superior Politécnica de Chimborazo (Riobamba, Ecuador). Currently working as Professor at that institution. E-mail: ivan.santillan@espoch.edu.ec 
In his research, Velásquez (2009) states the following:

Es importante fortalecer la cultura propia de la organización, ya que gracias a ella se crea un grupo de valores, conocimientos y reglas implícitas que rigen el comportamiento diario en el lugar de trabajo y favorece directamente la comunicación entre los miembros de la empresa, creando en los empleados un sentido de pertenencia con el mismo, además con ayuda de una cultura organizacional bien establecida y una estrategia de comunicación interna se obtendrán mejores resultados a nivel interior de la empresa. [It is important to strengthen the organization's culture, as it creates a set of values, knowledge and implicit rules governing daily behavior in the workplace and directly favors communication between company members, creating a sense of belonging in employees. In addition, better results will be obtained within a company with the help of a well-established organizational culture and an internal communication strategy.] (p. 32)

Similarly, Robbins and Judge (2009) state the following:

Organizational culture shows how employees perceive the characteristics of an organization's culture, not whether they like themthat is, it's a descriptive term. This is important because it differentiates culture from job satisfaction. (p. 552)

Regarding work environment and human talent management, Velásquez (2009) states the following:

El clima laboral es el medio ambiente humano y físico en el que se desarrolla el trabajo cotidiano, influye en la satisfacción y por lo tanto en la productividad. Está relacionado con el "saber hacer» del directivo con los comportamientos de las personas, con su manera de trabajar y de relacionarse, con su interacción con la empresa, con las máquinas que se utilizan y con la propia actividad de cada uno. [Work climate is the human and physical environment in which daily work takes place, influencing satisfaction and therefore productivity. It is related to the "know-how" of the manager in terms of people's behavior, the way they work and relate to each other, their interaction with the company, the machines they use and their own activities.] (p. 37)
Chiavenato (2002) says the following:

El Área de Recursos Humanos (ARH) es una de las áreas más afectadas por los recientes cambios que acontecen en el mundo moderno. Las empresas se dieron cuenta de que las personas son el elemento central de su sistema nervioso porque introducen la inteligencia en los negocios y la racionalidad en las decisiones. Tratar a las personas como recursos organizacionales es desperdiciar el talento y la mente productiva. Por tanto, para proporcionar esta nueva visión de las personas, hoy se habla de gestión del talento humano y no de recursos humanos, y ya no se tratan como empleados remunerados en función de la disponibilidad de tiempo para la organización, sino como socios y colaboradores del negocio de la empresa. [The Human Resources Department (HRD) is one of the areas most affected by recent changes in the modern world. Companies realized that people are the key element of their nervous system because they introduce intelligence into business and rationality into decisions. To treat people as organizational resources is to waste talent and productive minds. Therefore, in order to provide this new vision of people, today we speak about human talent management rather than human resources, and they are no longer treated as paid employees depending on the availability of time for the organization, but as partners and collaborators of the company.] (p. 23)

For Tejada (2003), "la gerencia del talento humano es la que señala que la dirección y la gestión en la organización se basan en el manejo efectivo de las potencialidades que tienen las personas" [human talent management indicates that direction and management in an organization are based on the effective management of people's potential] ( $p$. 117). Finally, García (2009) states as follows:

Una organización adquiere una ventaja competitiva trascendental cuando dedica parte de sus actividades, recursos y tiempo a la formación y gestión eficiente de su talento humano. Por ello, es necesario que el área de la empresa encargada de la gestión humana tenga claramente definidos los procesos y actividades. [An organization acquires a significant competitive advantage when it devotes part of its activities, resources and time to the training and efficient management of its human talent. Therefore, it is necessary that the area of the company in charge of 
human management has clearly defined processes and activities.] (p. 164)

Competency-based management of human talent is understood as a complex structure of attributes necessary for performance in specific situations. These attributes are character, attitudes, values, knowledge of the performed activity and any personal characteristics that can be reliably identified and demonstrated among workers who maintain excellent performance or among workers who are effective and ineffective. The aim of this research study is to propose a new concept of human competency in the workplace for the Ciudad del Auto (CIAUTO) company in Ambato, Ecuador; as well as to demonstrate the significance of human talent in achieving high company productivity, especially in the painting department.

In this context, this research conducted at the CIAUTO vehicle painting department addressed current issues from a theoretical-practical perspective, starting from strategic planning of human talent management, development of recruitment strategies, selection of skilled workforce with a correct description and role and job analysis. Later, location and training are addressed, keeping in mind the process of employee performance, low incentives and compensation, industrial health and safety, and quality of life. A pre-experimental study with a statistical analysis of a pre and posttest was also conducted with all analysis being framed within the context of Ecuadorian labor law.

\section{Competencies}

According to the International Labour Organization (ILO), competency is defined as the effective capacity to successfully carry out a fully identified work activity. For its part, the International Organization for Standardization (ISO) 9000 (2000), in the section regarding vocabulary and terms related to competency, defines competency as the "demonstrated ability to apply knowledge and skills" (p.19). Therefore, competency is a combination of characteristics specific to a person (attitudes, know-how, abilities, skills), expertise that makes it possible to successfully carry out any activity. In conclusion, the definition of the term "competency" has been key in the designing of the model, since it is based on the recognition of personal abilities.

\section{The Iceberg Model of Competencies}

Spencer and Spencer (1993) define the iceberg model on two levels. On one level, they suggest that people's knowledge and skills are easy to observe (visible) and are developed with training; while on another there is a hidden part that corresponds to relative competencies related to the motivation, attitudes and values that a person possesses and which determine personality, so that this is the most difficult part to modify or change. Therefore, at the time of recruitment, academic training is not sufficient, as it is necessary to evaluate other key aspects that may have a positive or negative impact on work performance according to the objectives set by the company or organization.

\section{Delphi Method}

This tool has been commonly used in prospective studies; Linstone and Turoff (1975) state that it "may be characterized as a method for structuring a group communication process so that the process is effective in allowing a group of individuals, as a whole, to deal with a complex problem" (p. 3).

It is distinguished by the anonymity of the individuals who make up the discussion group. Moreover, iteration and feedback are controlled, responses are provided in statistical form and there is heterogeneity within responses. In addition, the Delphi method consists of five stages: definition of objectives, selection of experts, panel conformation, development and launch of questionnaires, and exploitation of results.

\section{DESIGN OF ROLES AND JOB ANALYSIS}

Chiavenato (2002) defines role as the description of the activities performed by a person, the analysis and design of these; meanwhile, Gómez-Mejía, Balkin and Cardy (2008) state the following:

El diseño del puesto de trabajo es el proceso de organización del trabajo en tareas necesarias para llevarlo a cabo. Hay distintos enfoques del diseño del trabajo: la simplificación del trabajo, la ampliación del trabajo, la rotación entre trabajos, el enriquecimiento del trabajo y el diseño del trabajo en función de los equipos. Análisis del puesto de trabajo es el proceso sistemático de recopilación de información para tomar decisiones relativas al trabajo. El análisis del puesto de trabajo identifica tareas, obligaciones y responsabilidades de un determinado trabajo. [The design of the position is the process of organizing work into tasks necessary to carry it out. There are different approaches to work design: the simplification of work, the extension of work, the rotation between jobs, the enrichment of work and the design of work 
according to teams. Job analysis is the systematic process of gathering information to make decisions about work. Job analysis identifies tasks, duties and responsibilities of a particular job.] (p. 99)

In this context, it can be concluded that "el empleado proporciona conocimientos, habilidades, capacidades y destrezas, junto con su aptitud hacia el aprendizaje para alcanzar un desempeño laboral óptimo; la organización debe facilitarle una gestión, donde ese trabajador pueda incrementar esas cualidades para su rendimiento eficiente" [the employee provides knowledge, skills, abilities and expertise, together with his ability to learn in order to achieve optimal work performance; the organization must provide employees a type of management that can enhance those qualities for efficient performance] (Leal \& Sánchez, 2006, p. 542). In the same sense, Pineda, Pérez and Arango (2012) indicate that "el rendimiento por contrato fue el indicador base para medir la productividad. Un contrato es una serie de máquinas asignadas a un trabajador; el indicador de rendimiento es la relación entre la producción real y la esperada" [contract performance was the basic indicator for measuring productivity. A contract is a series of machines assigned to a worker; the performance indicator is the relationship between actual and expected production] (p. 38). Sherman, Bohlander and Snell (1999), as well as Rue and Byars (2000) state that the design defines how the tasks are to be performed, who will perform them and where.

\section{Human Talent Management Model}

Human talent is defined by Chiavenato (2009) as "el área que construye talentos por medio de un conjunto integrado de procesos, y que cuida al capital humano de las organizaciones, dado que es el elemento fundamental de su capital intelectual y la base de su éxito" [the area that builds talent through an integrated set of processes, and that cares for organizational, since it is the fundamental element of their intellectual capital and the basis of their success] (p. 9). Meanwhile, Eslava (2004) affirms that human talent management:

Es un enfoque estratégico de dirección cuyo objetivo es obtener la máxima creación de valor para la organización, a través de un conjunto de acciones dirigidas a disponer en todo momento del nivel de conocimiento, capacidades y habilidades en la obtención de los resultados necesarios para ser competitivos en el entorno actual y futuro. [is a strategic management approach whose objecti- ve is to obtain the maximum value creation for the organization, through a set of actions aimed at using knowledge, capacities and skills at all times in order to obtain the necessary results to be competitive in the current and future environment.] (p. 86)

Solano (2010) contributes to the definition indicating that human talent management models:

Establecen los requerimientos mínimos que, al ser cubiertos, pueden conducir a la satisfacción de los clientes, con la gestión de la calidad, al bienestar de las personas en el sitio de trabajo, con la gestión de la seguridad y salud, a reducir la contaminación, con la implementación de un sistema de gestión ambiental. [establish minimum requirements that, when fulfilled, can lead to customer satisfaction, through quality management; can lead to the well-being of people in the workplace, through safety and health management; can reduce pollution, through the implementation of an environmental management system.] (p. 84)

Thus, the basic components of the management model are: initial diagnosis, legal requirements, establishment of significant aspects, establishment of work areas, selection of managers, work instructions, control of corrective actions, procedures and guidelines.

\section{Competency measurement}

Skills development can be measured before and after the implementation of development plans and, in this way, the results can be compared and the appropriate conclusions drawn. One such methodology is that proposed by Alles (2005) who proposes to first measure desired or required skills to then assess and measure them in the first and second year. If the competencies are close to requirements, then they have been effective.

Behavior change is calculated using the following formula:

$$
C_{A p}=\frac{\operatorname{Ap}(\mathrm{d})}{\operatorname{Ap}(\mathrm{a})}
$$

Where:

$C_{A p}=$ observable change in skills as a result of training

$A p(d)=$ skills demonstrated after on-the-job training 
$\mathrm{Ap}(\mathrm{a})=$ skill level existing prior training, using the same measurement criteria

Data used for the calculations is obtained and gathered through questionnaires, interviews and observation.

\section{METHODOLOGY}

This is a pre-experimental study, as according to Hernández, Fernández and Baptista (2014) it serves as an exploratory study with pretest/posttest conducted on a single group. It is also a descriptive, applicative and field study, where contact with electrophoresis workstations (ELPO painting technique) is made-using a methodical procedure of data collection, analysis and formal presentation, supported by direct data collection from the CIAUTO company in Ambato (Ecuador) - in order to analyze the sealing process and first top-coat paint process. In addition, the Delphi method was used to compile the judgments issued by the company's expert panel: the general manager, human talent coordinator, welding area leader, painting area leader, financial coordinator and operators, all this by means of a questionnaire and interview.

\section{Competency-based human talent management process}

Competency-based human talent management in the CIAUTO vehicle painting plant in Ambato, Ecuador, consists of the following subsystems: recruitment and selection, training and performance evaluation. See Figure 1.

\section{Associated documents and records}

The documents available to the company for the selection, recruitment, training and evaluation of the personnel to carry out the activities at the workstation were analyzed, as these make it possible to carry out the processes and sub-processes of human talent management. These documents are:

- [SOP-06-PL-01] Training plan.

- [SOP-06-FR-01] Role description form.

- [SOP-06-FR-02] Job application and interview form.

- [SOP-06-FR-03] Documents requested for the employee file form.

- $\quad$ [SOP-06-FR-04] Induction form for the job.

- [SOP-06-FR-05] Training registration form.

- $\quad$ [SOP-06-FR-06] Staff assessment form (3 months).

- [SOP-06-FR-07] Staff assessment form (12 months).

- [SOP-06-FR-08] Performance assessment form (after all training).

\section{Job profile}

Profile identification is performed using the Iceberg Model of Competencies, in which two large blocks are proposed. The first includes skills that are easy to detect (skills, knowledge of basic sciences, mate-

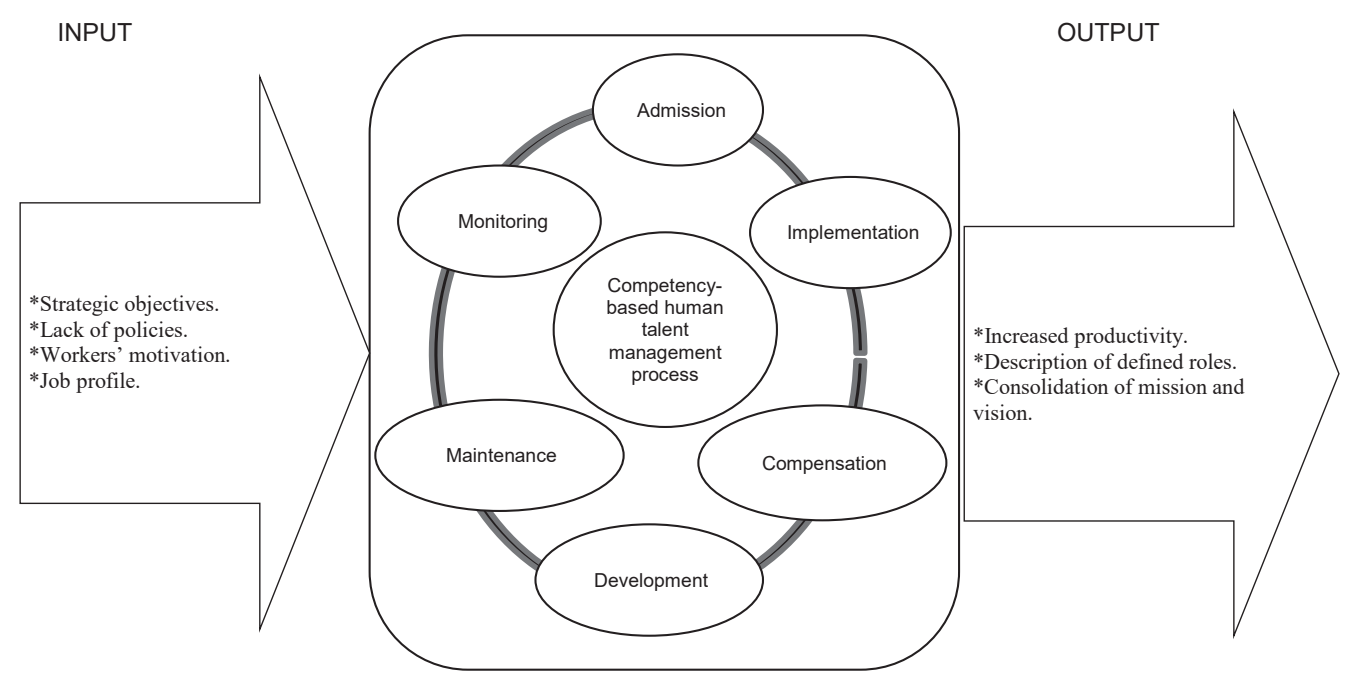

Figure 1. Competency-based human talent management. Source: Prepared by the authors. 
rials and painting). A direct questionnaire with open questions designed to know the activities of each role is also presented. The second block includes skills that are difficult to detect, therefore employees' competencies and attitudes towards the company are evaluated with testing.

\section{Responsibility and authority}

The human talent coordinator is responsible for the implementation, maintenance and improvement of the model. The coordinator is also responsible for developing and coordinating recruitment and selection activities, training, performance evaluation and effectiveness of the organization's staff training.

\section{Survey}

The survey focuses on measuring employee's attitudes using a standard response format, according to the Likert method, as shown in the table1.

\section{QUESTIONNAIRE}

Objective: Identify the attitude or behavior of employees during the performance of their work activities.

Mark with an $\mathrm{X}$ the answer you consider correct according to the question ( 5 = very satisfied, $4=$ satisfied, 3 = moderately satisfied, 2 = unsatisfied, 1 = very unsatisfied).

A deductive method was used in this research. The information obtained using data gathering tools was tabulated by means of Excel and SPSS Statistics 21 software, while the hypothesis was tested with the Chi-square method using, with data before and after implementation of improvements.

\section{Population and sample}

The population of the study consisted of eight employees, which is the entire CIAUTO company painting department.

The survey was subjected to a reliability statistics analysis obtaining a Cronbach's alpha of 0.627 , which indicates that the test applied has an acceptable level of reliability.

\section{RESULTS AND DISCUSSION}

Surveys were carried out to obtain the parameters that enable the improvement of the company's goals. To achieve a correct management of human talent, each production process was measured. The results are shown in the following table:

Table 2. Productivity rate before implementation of improvements.

\begin{tabular}{|c|c|c|c|}
\hline Day & $\begin{array}{c}\text { Production } \\
\text { (vehicles) }\end{array}$ & Time (hours) & $\begin{array}{c}\text { Productivity } \\
\text { rate }\end{array}$ \\
\hline 1 & 4 & 7.50 & 0.533 \\
\hline 2 & 5 & 8.00 & 0.625 \\
\hline 3 & 4 & 8.00 & 0.500 \\
\hline 4 & 5 & 8.00 & 0.625 \\
\hline 5 & 4 & 7.00 & 0.571 \\
\hline 6 & 4 & 7.50 & 0.533 \\
\hline 7 & 4 & 7.75 & 0.516 \\
\hline 8 & 5 & 7.50 & 0.667 \\
\hline 9 & 5 & 8.00 & 0.625 \\
\hline 10 & 5 & 8.00 & 0.625 \\
\hline 11 & 5 & 8.00 & 0.625 \\
\hline 12 & 5 & 7.75 & 0.645 \\
\hline 13 & 4 & 7.25 & 0.552 \\
\hline 14 & 5 & 8.25 & 0.606 \\
\hline 15 & 4 & 7.50 & 0.533 \\
\hline & Productivity rate average & 0.585 \\
\hline
\end{tabular}

Source: Prepared by the authors.

Table 1. Survey to measure employee's attitudes.

\begin{tabular}{|c|c|c|c|c|c|}
\hline QUESTION & 1 & 2 & 3 & 4 & 5 \\
\hline \multicolumn{6}{|l|}{ How do you feel about your job? } \\
\hline \multicolumn{6}{|l|}{ How do you rate your work environment? } \\
\hline \multicolumn{6}{|l|}{ Are you satisfied with the attitude of your immediate boss? } \\
\hline \multicolumn{6}{|l|}{ How satisfied are you with the way you are treated by the company? } \\
\hline \multicolumn{6}{|c|}{$\begin{array}{l}\text { Are you satisfied with the working conditions the company provides you with for the accompli- } \\
\text { shment of tasks? }\end{array}$} \\
\hline \multicolumn{6}{|l|}{ Are you satisfied with the impartiality of your superiors? } \\
\hline \multicolumn{6}{|l|}{ How do you feel about the freedom they give you to do your job? } \\
\hline \multicolumn{6}{|l|}{ Are you satisfied with the customer service the company provides clients? } \\
\hline \multicolumn{6}{|l|}{ Are you satisfied with the treatment you receive within the company? } \\
\hline How satisfied are you with the processes and procedures within the company? & & & & & \\
\hline
\end{tabular}

Source: Prepared by the authors. 
Table 2 shows that the productivity rate is 0.585 vehicles per hour, thus determining that a vehicle is painted every 1 hour and $42 \mathrm{~min}$. Therefore, for an eight-hour workday, productivity is 4.68 vehicles per day.

Subsequently, a survey to identify the attitude of workers in their workplace, using the questionnaire in Table 1, was conducted on November 15, 2016. The result showed that $38.75 \%$ of employees are moderately satisfied with their jobs. See the following figure:

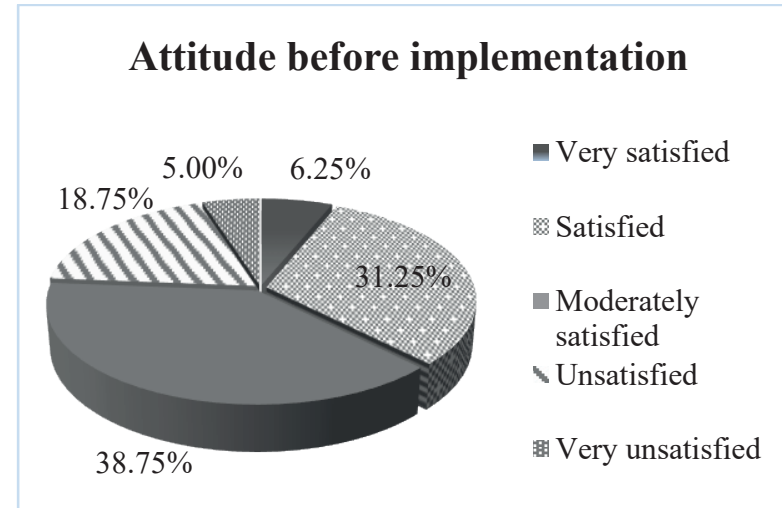

Figure 2. Frequency of employee's attitude as of November 15, 2016.

Source: Prepared by the authors.

Despite being a pre-experimental type study, the survey addressed to painting department employees was carried out three months after implementing the improvements, in order to determine the changes in employees' attitudes towards the company. The results demonstrate that $46.25 \%$ were satisfied with their jobs and conditions. See the following figure:

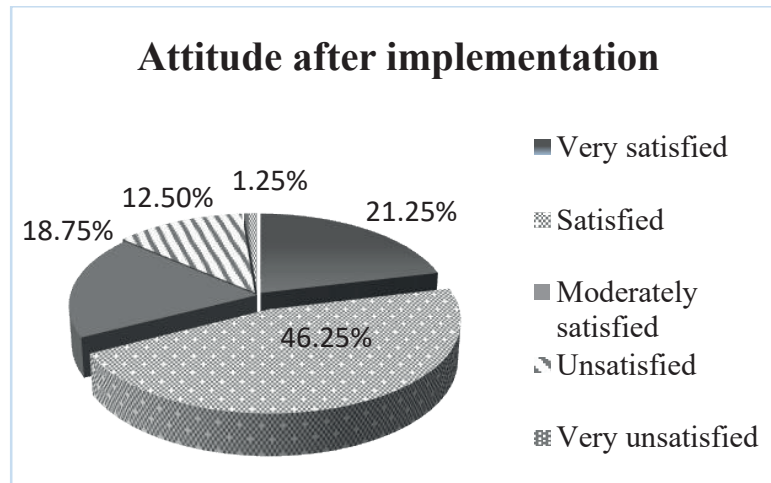

Figure 3. Frequency of employee's attitude as of March 1, 2017 (posttest).

Source: Prepared by the authors.
The productivity rate was measured after implementation of improvements, obtaining the data shown in Table 3.

Table 3. Productivity rate after implementation of improvements.

\begin{tabular}{|c|c|c|c|}
\hline Day & $\begin{array}{c}\text { Production } \\
\text { (vehicles) }\end{array}$ & Time (hours) & $\begin{array}{c}\text { Productivity } \\
\text { rate }\end{array}$ \\
\hline 1 & 7 & 8 & 0.875 \\
\hline 2 & 7 & 8 & 0.875 \\
\hline 3 & 8 & 8 & 1.000 \\
\hline 4 & 8 & 8 & 1.000 \\
\hline 5 & 8 & 8 & 1.000 \\
\hline 6 & 8 & 8 & 1.000 \\
\hline 7 & 7 & 8 & 0.875 \\
\hline 8 & 7 & 8 & 0.875 \\
\hline 9 & 7 & 8 & 0.875 \\
\hline 10 & 8 & 8 & 1.000 \\
\hline 11 & 7 & 8 & 0.875 \\
\hline 12 & 7 & 8 & 0.875 \\
\hline 13 & 7 & 8 & 0.875 \\
\hline 14 & 7 & 8 & 0.875 \\
\hline 15 & 8 & 8 & 1.000 \\
\hline & \multicolumn{2}{|c|}{ Productivity rate average } & 0.925 \\
\hline
\end{tabular}

Source: Prepared by the authors.

Table 3 shows that the productivity rate is 0.925 vehicles per hour and 7.4 vehicles per day for an eight-hour workday.

In Figures 3 and 4 regarding attitudes before and after implementation of improvements, it is observed that there is a change in the employees' attitudes towards the company, as the number of very satisfied employees has increased from $6.25 \%$ to $21.25 \%$ and satisfied employees increased from $31.25 \%$ to $46.25 \%$. There the number of employees who feel moderately satisfied decreased from $38.75 \%$ to $18.75 \%$; unsatisfied employees, from $18.75 \%$ to $12.5 \%$; and very unsatisfied from $5 \%$ to $1.25 \%$.

To verify the change in workers' behavior, in particular regarding the "satisfactory" rate, equation (1) is used, obtaining 1.48 (a value greater than 1 is considered a positive change in attitudes). This is shown in Tables 2 and 3, where the average productivity rate increased from 0.585 -before applying competency-based human talent management- - to 0.925 .

To determine that the objective was achieved, the null hypothesis $(\mathrm{Ho})$ and alternative hypothesis $(\mathrm{Ha})$ were used.

Ho: Competency-based human talent management, in the CIAUTO company painting department, does 
Tabla 4. Observed and expected frequencies.

\begin{tabular}{|l|c|c|c|c|}
\hline \multirow{2}{*}{\multicolumn{1}{c|}{ Variable }} & \multicolumn{2}{c|}{ Observed frequencies } & \multicolumn{2}{c|}{ Expected frequencies } \\
\cline { 2 - 5 } & Before (Px) & After (Py) & Before & After \\
\hline Very satisfied & 5 & 17 & 10.83 & 11.17 \\
\hline Satisfied & 25 & 37 & 30.51 & 31.49 \\
\hline Moderately satisfied & 31 & 15 & 22.64 & 23.36 \\
\hline Unsatisfied & 15 & 10 & 12.30 & 12.70 \\
\hline Very unsatisfied & 4 & 1 & 2.46 & 2.54 \\
\hline Productivity & 4.68 & 7.4 & 5.95 & 6.14 \\
\hline Subtotal & 84.68 & 87.4 & & \\
\hline
\end{tabular}

Source: Prepared by the authors.

Tabla 5. Chi-square test.

\begin{tabular}{|l|c|c|c|}
\hline & Value & Degrees of freedom & Asymptotic sig. (2-sided) \\
\hline Pearson's Chi-square & $17.546^{\mathrm{a}}$ & 5 & 0.004 \\
\hline Likelihood-ratio & 18.183 & 5 & 0.003 \\
\hline Linear-by-linear association & 11.265 & 1 & 0.001 \\
\hline No. of valid cases & 172 & & \\
\hline
\end{tabular}

Source: Prepared by the authors.

a. Two cells $(16.7 \%)$ have an expected count less than 5 . The minimum expected count is 2.47 .

not enable the improvement of its processes and procedures and decreases productivity.

Ha: Competency-based human talent management, in the CIAUTO company painting department, enables the improvement of its processes and procedures and increases productivity.

The following was proposed for verification: $\mathrm{Ho}: \mathrm{Py}$ = Px; Ha: Py > Px. To do so, the data was tabulated in the following table:

A Chi-square value of 11.07 was obtained, with an error margin of $5 \%$ and 5 degrees of freedom. Subsequently, the hypothesis was accepted using the software SPSS Statistics 21 . See the following table:

Finally, in Table 5 it is observed that the calculated Chi-square is $17.55(p<0.05)$, with which the null hypothesis is rejected and the alternate hypothesis $(\mathrm{Ha})$ is accepted; thus, competency-based human talent management in the CIAUTO company painting department enables the improvement of its processes and procedures and increases productivity according to the research conducted.

\section{CONCLUSIONS}

1. The Competency-based Human Talent Management Model in the CIAUTO company vehicle painting department, in Ambato (Ecuador), is defined by the processes of admission, preservation and monitoring of personnel. Therefore, the analysis and design of roles are performed using the Delphi method and the activities to be carried out by each worker are described. Thus, for skill measurement, the situation before and after implementation of the improvement plans must be considered. They should then be evaluated using the Likert scale, applying performance measurement strategies based on competency-based management indicators.

2. The procedure for competency-based human talent management in the CIAUTO company vehicle painting department is determined by means of the processes of admission, application, compensation, development, maintenance and monitoring of personnel. Also, documents and associated records are established through the analysis of the plant's requirements, people responsible for the implementation, maintenance and improvement of processes.

3. The procedure of competency-based human talent management model strengthens human talent in the CIAUTO company vehicle painting department, as a means to achieve effectiveness and efficiency, generated from greater competitiveness of human resources, which establishes the process and ensures staff training. In addition, an adequate job description ensures the correct selection and placement of 
staff and, as a consequence, results in employee retention, leading the company to success.

4. Although it is a pre-experimental type study, statistical analysis was used, and a value of 1.48 was obtained after applying competency measurement with a pre and posttest, identifying that there was a positive transformation in attitude. The company has motivated and efficient workers, who receive permanent training and are paid according to their performance, skills and abilities, which results in an increase in productivity from 4.68 to 7.4 vehicles per day.

5. The implementation competency-based human talent management policy in the CIATO company vehicle painting department enables the improvement of its processes and procedures and increases productivity.

\section{REFERENCES}

[1] Alles, M. (2005). Desempeño porcompetencias. Evaluación $360^{\circ}$. Buenos Aires, Argentina: Ediciones Granica. Retrieved from https:// www.academia.edu/22501484/Alles-MarthaDesempeño-por-competencias-de-360.

[2] Chiavenato, I. (2002). Gestión del talento humano. Bogotá, Colombia: McGraw-Hill.

[3] Chiavenato, I. (2009). Gestión del talento humano. México, D. F., Mexico: McGraw-Hill.

[4] Eslava, E. (2004). Gestión del talento humano en las organizaciones. Retrieved from https:// es.scribd.com/document/114171987/GestionTalento-Humano-Organizaciones-Libro.

[5] García, M. (2009). Los macro-procesos: un nuevo enfoque en el estudio de la gestión humana. Pensamiento y Gestión, (27), 162-200.

[6] Gómez-Mejía, L.; Balkin, D. \& Cardy, R. (2008). Gestión de recursos humanos. Madrid, Spain: Pearson Educación S. A.

[7] Hernández, R.; Fernández, C. \& Baptista, M. (2014). Metodología de la investigación. México, D. F., Mexico: McGraw-Hill.

[8] Leal, R. \& Sánchez, N. (2006). Competencias profesionales del personal operativo de las empresas automotrices para su eficiente desempeño laboral. Revista Venezolana de Ciencias Sociales, 10(2), 541-560.

[9] Linstone, H. A. \& Turoff, M. (Eds.) (1975). The Delphi method. Techniques and applications.
Retrieved from https://web.njit.edu/ turoff/ pubs/delphibook/delphibook.pdf.

[10] Organización Internacional de Normalización (ISO) 9000 (2000). Norma internacional. Sistemas de gestión de la calidad: conceptos y vocabulario. Retrieved from https:// gestiondecalidadmpn.files.wordpress. com/2012/02/iso-9000-2000-sistemasde-gestic $3 b 3 n-d e-l a-c a l i d a d-c o n c e p t o s-y-$ vocabulario.pdf.

[11] Pineda, U.; Pérez, G. \& Arango, M. (2012). Medición del impacto de las competencias laborales en la productividad de los procesos: caso de una empresa manufacturera. Innovar. Revista de Ciencias Administrativas y Sociales, 22(45), 37-50. Retrieved from https://www. redalyc.org/pdf/818/81824864004.pdf.

[12] Robbins, S. \& Judge, T. (2009). Comportamiento organizacional. México, D. F., Mexico: Pearson Educación.

[13] Rue, L. \& Byars, L. (2000). Management, Skills and Applications. Boston, USA: Irin / McGraw-Hill.

[14] Saldarriaga, J. G. (2009). La responsabilidad social aplicada como estrategia de gestión humana. Retrieved from http://www.ascolfa. edu.co/web/archivos/conferencia2009/ memorias/MemoriasCladea2009/upac01_ submission_411.pdf.

[15] Sherman, A.; Bohlander, G. \& Snell, S. (1999). Administración de recursos humanos. México, D. F., Mexico: Thomson Editores.

[16] Solano, P. (2010). Modelos de gestión ambiental aplicados en la Compañía Ecuatoriana de Caucho S. A. (Master's thesis). Universidad de Cuenca, Cuenca.

[17] Spencer, L. \& Spencer, P. (1993). Competence at work: models for superior performance. New York, USA: John Wiley \& Sons Inc.

[18] Tejada, A. (2003). Los modelos actuales de gestión en las organizaciones. Gestión del talento, gestión del conocimiento y gestión por competencias. Psicología desde el Caribe, (12), 115-133. Retrieved from https://www. redalyc.org/pdf/213/21301208.pdf.

[19] Velásquez, M. (2009). Propuesta de planeación estratégica para la gestión del talento humano de la sección agencia de viajes del departamento comercial de la subdirección de recreación de CAFAM. (Undergraduate thesis). Universidad La Salle, Arequipa. 